\title{
Comparison of diagnostic methods for diabetes mellitus based on prevalence of retinopathy in a Japanese population: the Hisayama Study
}

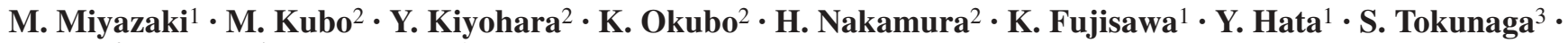 \\ M. Iida ${ }^{2} \cdot$ Y. Nose $^{4} \cdot$ T. Ishibashi ${ }^{1}$ \\ ${ }^{1}$ Department of Ophthalmology, Graduate School of Medical Sciences, Kyushu University, Fukuoka, Japan \\ ${ }^{2}$ Department of Medicine and Clinical Science, Graduate School of Medical Sciences, Kyushu University, Fukuoka, Japan \\ ${ }^{3}$ Department of Preventive Medicine, Graduate School of Medical Sciences, Kyushu University, Fukuoka, Japan \\ ${ }^{4}$ Department of Medical Information Science, Graduate School of Medical Sciences, Kyushu University, Fukuoka, Japan
}

\section{Abstract}

Aims/hypothesis. The aims of this study were to compare the ability of tests measuring fasting plasma glucose, 2-h plasma glucose and $\mathrm{HbA}_{1} \mathrm{c}$ levels in predicting specific diabetic retinopathy, and to determine the cut-off level of each measurement for diagnosing diabetes in a Japanese population.

Methods. In a total of 1637 subjects, fasting plasma glucose, 2-h plasma glucose and $\mathrm{HbA}_{1} \mathrm{c}$ levels were measured in a 75-g oral glucose tolerance test, and diabetic retinopathy was assessed by ophthalmic examination. We calculated receiver operating characteristic (ROC) curves as well as the prevalence of diabetic retinopathy by deciles of the distribution of these glycaemic measurements.

Results. Of the subjects, 37 (2.3\%) had diabetic retinopathy. The prevalence of retinopathy dramatically increased in the tenth decile of each variable. Analysis with ROC curves showed that the optimal cut-off lev- els for diagnosis of diabetes were $6.4 \mathrm{mmol} / \mathrm{l}$ for fasting plasma glucose, $11.1 \mathrm{mmol} / \mathrm{l}$ for 2 -h plasma glucose, and $5.7 \%$ for $\mathrm{HbA}_{1} \mathrm{c}$. The sensitivities for the cut-off point of the three measurements were identical $(86.5 \%)$, and the specificities were similar (fasting plasma glucose $87.3 \%$; 2-h plasma glucose $89.6 \%$; $\mathrm{HbA}_{1} \mathrm{c} 90.1 \%$ ). The area under the ROC curve for 2-h plasma glucose $(96.1 \%)$ was slightly but not significantly larger than that for fasting plasma glucose $(90.0 \%)$ and that for $\mathrm{HbA}_{1} \mathrm{c}(94.5 \%)$.

Conclusions/interpretation. Our findings suggest that measuring fasting plasma glucose or $\mathrm{HbA}_{1} \mathrm{c}$ is just as useful as measuring 2-h plasma glucose for the diagnosis of diabetes, and that the cut-off point for diagnostic fasting plasma glucose level is lower than that of the current diagnostic criteria.

Keywords Diabetic retinopathy $\cdot$ Fasting plasma glucose $\cdot \mathrm{HbA}_{1} \mathrm{c} \cdot$ Receiver operating characteristic curve
Received: 10 February 2004 / Accepted: 15 April 2004

Published online: 28 July 2004

C) Springer-Verlag 2004

M. Miyazaki (- $)$

Department of Ophthalmology,

Graduate School of Medical Sciences, Kyushu University,

3-1-1 Maidashi, Higashi-ku, Fukuoka 812-8582, Japan

E-mail: miho-m@med.kyushu-u.ac.jp

Tel.: +81-92-6425881, Fax: +81-92-6425889

Abbreviations: ADA, American Diabetes Association . FPG, fasting plasma glucose $\cdot 2$-h PG, 2-hour post-load plasma glucose $\cdot$ NHANES III, the Third National Health and Nutrition Examination Survey $\cdot$ ROC, receiver operating characteristic

\section{Introduction}

In 1997 the Expert Committee of the American Diabetes Association (ADA) proposed new revised criteria for diagnosing diabetes [1]. These criteria lowered the diagnostic fasting plasma glucose (FPG) from $\geq 7.8 \mathrm{mmol} / 1$ to $\geq 7.0 \mathrm{mmol} / 1$, but kept the 2-hour postload plasma glucose (2-h PG) value at $\geq 11.1 \mathrm{mmol} / 1$ [1]. The WHO subsequently endorsed these recommendations [2]. These diagnostic levels were determined based on the findings of several populationbased studies, including those of Pima Indians [3], the Third National Health and Nutrition Examination Survey (NHANES III) participants [1], and Egyptians [4]. However, the sensitivity and tolerability to glucose 
load have been reported to vary among ethnic groups $[5,6,7]$, and little information has been made available for the Japanese population [8]. In the present article, therefore, we compared the efficacy of tests for FPG, 2-h PG and $\mathrm{HbA}_{1} \mathrm{c}$ levels in predicting specific diabetic retinopathy in a Japanese population, and determined the cut-off levels by separating the subjects who were at substantially increased risk of diabetic retinopathy from those who were not.

\section{Subjects and methods}

Study population. The Hisayama study is an ongoing prospective cohort study on cardiovascular disease and its risk factors in a community of Hisayama Town adjoining Fukuoka City, a metropolitan area in southern Japan. The enrolment criteria, characteristics of the study population, and overall design of this study have been described in detail in previous studies [9, $10,11]$. As part of the follow-up survey in 1998, we performed a cross-sectional examination, including a 75-g OGTT and ophthalmic examination, of Hisayama residents aged 40 to 79 years. Of a total of 3847 residents in that age group, 1950 subjects $(50.7 \%)$ consented to participate in the study. After excluding 41 subjects who had already eaten breakfast at the examination, three who were on insulin therapy, 182 who underwent the examination at home, and 87 in whom gradable fundus photographs could not be obtained, a total of 1637 individuals (637 men and 1000 women) successfully completed the 75-g OGTT and ophthalmic examination.

Laboratory measurements. Blood samples were collected from an antecubital vein after an overnight fast for the determination of plasma glucose and $\mathrm{HbA}_{1} \mathrm{c}$ levels. After the fasting blood specimen had been taken, the OGTT was performed with a 75-g glucose equivalent carbohydrate load (Trelan G; Shimizu Pharmaceutical, Shimizu, Japan) between 08.00 and 10.30 hours. Subjects receiving oral hypoglycaemic agents omitted their medication until the OGTT. At $120 \mathrm{~min}$ after ingestion of the solution, a blood sample was obtained for the determination of post-loading plasma glucose levels. These specimens were analysed within $24 \mathrm{~h}$. Plasma glucose was determined by the glucose-oxidase method, and $\mathrm{HbA}_{1} \mathrm{c}$ was measured by a high-pressure lipid chromatographic assay.

Ophthalmic examination and classification of diabetic retinopathy. Each participant underwent comprehensive ophthalmic examination, including stereoscopic fundus examination using indirect ophthalmoscopy, and examination with a slit lamp biomicroscope with a "superfield lens" (Volk, Mentor, Ohio, USA) after pupil dilatation. Fundus photographs $\left(45^{\circ}\right)$ were taken using a Topcon "non-mydriatic" TRC NW-5 fundus camera (Topcon, Tokyo, Japan) and Fujichrome slide film (Sensia II; Fujifilm, Tokyo, Japan). The presence of diabetic retinopathy was determined based on the grading of fundus examinations by indirect ophthalmoscopy, slit lamp, and colour fundus photographs. The photographs were graded by masked photo graders using a modification of the Airlie House classification system: (i) no retinopathic changes; (ii) mild non-proliferative retinopathy; (iii) moderate retinopathy; and (iv) proliferative retinopathy $[12,13,14,15]$. The degree of diabetic retinopathy was determined according to the grading in the worse eye.
Statistical analysis. The SAS computer package (SAS Institute, Cary, N.C., USA) and Stata version 8.0 (Stata, College Station, Tex., USA) were used to perform all statistical analyses. The sensitivity of a specific glycaemic cut-off point was defined as its ability to correctly identify individuals who have diabetic retinopathy, and its specificity was defined as its ability to correctly identify individuals who do not have diabetic retinopathy. To compare the ability of FPG, 2-h PG and $\mathrm{HbA}_{1} \mathrm{c}$ measurements to detect the presence or absence of retinopathy over a range of values, we calculated receiver operating characteristic (ROC) curves and compared the areas beneath them $[16,17]$. The diagnostic properties of specific cut-off levels of FPG, 2-h PG and $\mathrm{HbA}_{1} \mathrm{c}$ concentrations were defined by maximising the sensitivity and specificity to identify diabetic retinopathy. A two-sided $p$ value of less than 0.05 was considered statistically significant.

Ethical considerations. This study was approved by the Human Ethics Review Committee of Kyushu University Graduate School of Medical Sciences, and was carried out in accordance with the Declaration of Helsinki. Informed consent was obtained from all participants.

\section{Results}

Of the study participants, $37(2.3 \%)$ were found to have diabetic retinopathy. Mild non-proliferative retinopathy (category ii), moderate retinopathy (category iii) and proliferative retinopathy (category iv) were found in $27(1.6 \%), 5(0.3 \%)$ and $5(0.3 \%)$ subjects respectively. When the subjects were divided according to the ADA fasting glucose criteria, diabetic retinopathy was found in four subjects $(0.3 \%)$ with an FPG level of $<6.1 \mathrm{mmol} / \mathrm{l}$, in nine $(5.9 \%)$ with an FPG of 6.1 to $6.9 \mathrm{mmol} / \mathrm{l}$, and in $24(23.1 \%)$ with an FPG of $\geq 7.0 \mathrm{mmol} / \mathrm{l}$ (Table 1). Likewise, five subjects (1.8\%) with a 2-h PG level of 7.8 to $11.0 \mathrm{mmol} / \mathrm{l}$ and 32 (20.5\%) with a 2 -h PG of $\geq 11.1 \mathrm{mmol} / 1$ had some degree of retinopathy, but there was no subject with retinopathy in the group with a $2-\mathrm{h}$ PG of $<7.8 \mathrm{mmol} / \mathrm{l}$.

Figure 1 shows the prevalence of diabetic retinopathy by deciles of the distribution of the FPG, 2-h PG and $\mathrm{HbA}_{1} \mathrm{c}$ levels. All three measures of glycaemia were strongly associated with retinopathy, and the prevalence increased dramatically in the tenth decile of each variable, corresponding to an FPG of $\geq 6.5 \mathrm{mmol} / \mathrm{l}$, a $2-\mathrm{h} \mathrm{PG}$ of $\geq 11.0 \mathrm{mmol} / \mathrm{l}$, and $\mathrm{HbA}_{1} \mathrm{c}$ levels of $\geq 5.8 \%$. The prevalences of retinopathy in the tenth decile of the FPG, 2-h PG and $\mathrm{HbA}_{1} \mathrm{c}$ levels were $16 \%, 20 \%$ and $20 \%$ respectively, while those in the ninth decile were $3 \%, 2 \%$ and $2 \%$ respectively.

To compare the ability of each glycaemic measurement to predict the presence of diabetic retinopathy, we calculated the sensitivity and specificity, and plotted ROC curves. As shown in Figure 2, the area under the ROC curve for 2-h PG was $96.1 \%$ (95\% CI: 94.4-97.7) and was slightly but not significantly larger than that for FPG (90.0\%; 95\% CI: 83.8-96.7; $p=0.076)$ and that for $\mathrm{HbA}_{1} \mathrm{c}(94.5 \%$; 95\% CI: 91.6-97.5; $p=0.296)$. The cut-off level defined by the 
Table 1. The prevalence of diabetic retinopathy by fasting and 2-h plasma glucose levels defined by the 1997 ADA criteria, the Hisayama Study 1998

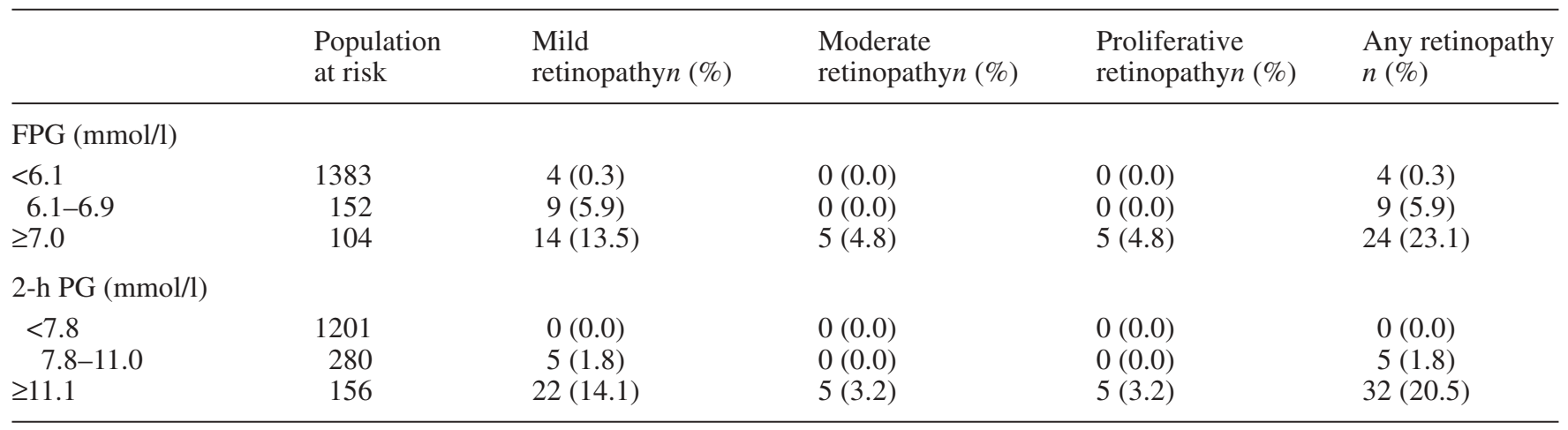

FPG, fasting plasma glucose; 2-h PG, 2-hour plasma glucose

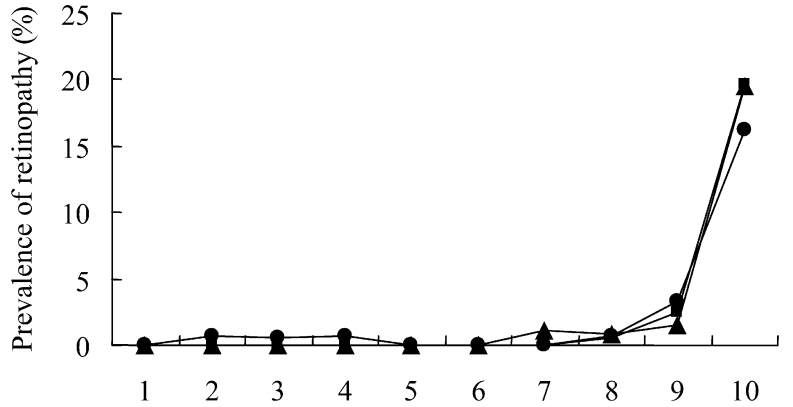

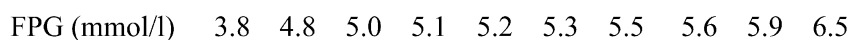

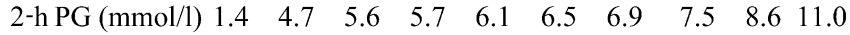
$\begin{array}{lllllllllll}\mathrm{HbA}_{1} \mathrm{c}(\%) & 4.1 & 4.7 & 4.8 & 4.9 & 5.0 & 5.1 & 5.2 & 5.3 & 5.5 & 5.8\end{array}$

Fig. 1. Prevalence of retinopathy by deciles of the distribution of FPG, 2-h PG and $\mathrm{HbA}_{1}$ c levels in the Hisayama study. The $x$ axis labels indicate the lower limit of each decile group. Black circles, FPG; black squares, 2-h PG; black triangles, $\mathrm{HbA}_{1} \mathrm{c}$

Table 2. Optimal cut-off points defined by maximising the sensitivity and specificity to identify diabetic retinopathy, the Hisayama Study 1998

\begin{tabular}{lrlc}
\hline & FPG & 2-h PG & $\mathrm{HbA}_{1} \mathrm{c}$ \\
\hline Cut-off point & $6.4 \mathrm{mmol} / 1$ & $11.1 \mathrm{mmol} / 1$ & $5.7 \%$ \\
Sensitivity (\%) & 86.5 & 86.5 & 86.5 \\
Specificity (\%) & 87.3 & 89.6 & 90.1 \\
ROC curve area (\%) & 90.0 & 96.1 & 94.5 \\
\hline
\end{tabular}

FPG, fasting plasma glucose; 2-h PG, 2-hour plasma glucose; $\mathrm{ROC}$, receiver operating characteristic

maximum of sensitivity and specificity was $6.4 \mathrm{mmol} / \mathrm{l}$ for FPG, $11.1 \mathrm{mmol} / \mathrm{l}$ for $2-\mathrm{h} \mathrm{PG}$, and $5.7 \%$ for $\mathrm{HbA}_{1} \mathrm{c}$ (Table 2). The sensitivities of these cut-off points for the three measurements were identical $(86.5 \%)$, and the specificities were similar (FPG $87.3 \%$; 2-h PG $89.6 \%$; $\mathrm{HbA}_{1} \mathrm{c} 90.1 \%$ ). The specificity for the FPG level of $7.0 \mathrm{mmol} / \mathrm{l}$ was higher $(91.3 \%)$, but its sensitivity $(70.3 \%)$ was lower than that for the FPG of $6.4 \mathrm{mmol} / \mathrm{l}$.

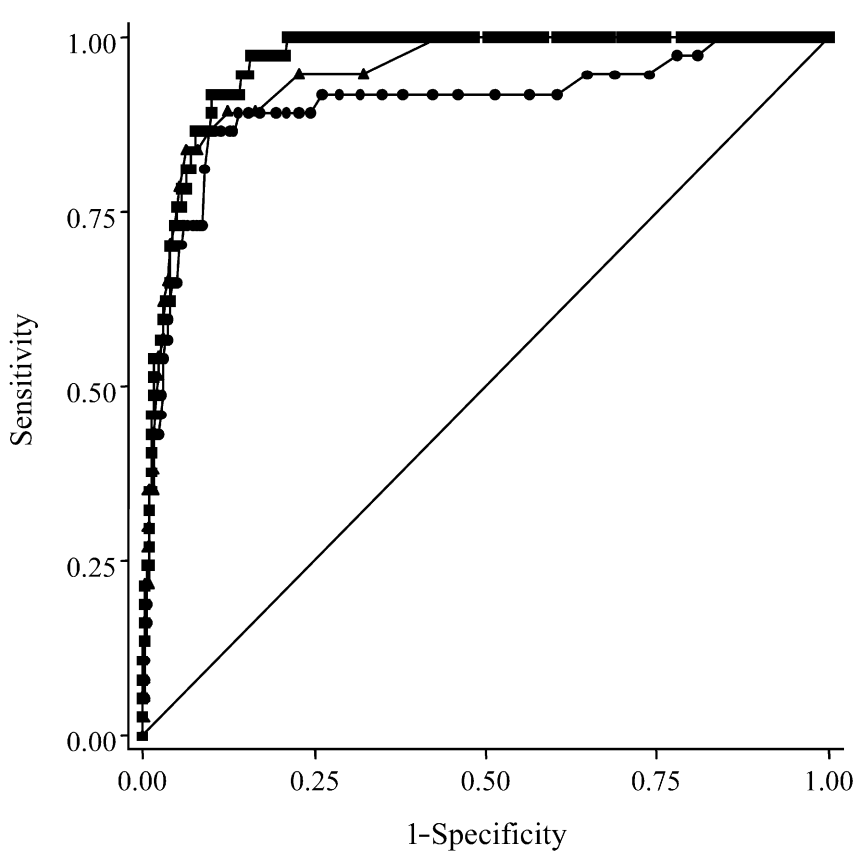

Fig. 2. Receiver operating characteristics (ROC) curves for FPG, 2-h PG and $\mathrm{HbA}_{1} \mathrm{c}$ measures for predicting the presence of diabetic retinopathy. Black circles, FPG (ROC area 90.0\%); black squares, 2-h PG (ROC area 96.1\%); black triangles, $\mathrm{HbA}_{1} \mathrm{c}$ (ROC area 94.5\%); black line, reference

\section{Discussion}

The current guidelines for the diagnosis of diabetes are based on several population studies examining the relationship between the measures of glycaemia and retinopathy [1]. The Hisayama study has allowed us to collect data on a large number of Japanese individuals with a range of glucose levels. We compared the efficacy of tests measuring FPG, 2-h PG and $\mathrm{HbA}_{1} \mathrm{c} \mathrm{lev-}$ els in predicting diabetic retinopathy, and found no significant difference in the ability to predict retinopathy among the three measures of glycaemia. In our subjects, the optimal cut-off levels defined by the ROC curves maximising the sensitivity and specificity 
to identify diabetic retinopathy were $6.4 \mathrm{mmol} / \mathrm{l}$ for FPG , $11.1 \mathrm{mmol} / 1$ for $2-\mathrm{h}$ PG, and $5.7 \%$ for $\mathrm{HbA}_{1} \mathrm{c}$. In addition, according to the prevalence of retinopathy examined by deciles of the distribution of the FPG, 2-h PG and $\mathrm{HbA}_{1} \mathrm{c}$ levels, a threshold was evident between the ninth and tenth deciles of each variable, below which retinopathy was almost absent and above which a distinct increase in retinopathy was observed. The cut-off point between the ninth and tenth deciles of each measure of glycaemia was very similar to that determined by the ROC curve. In contrast, the cut-off level of 2-h PG was consistent with the current ADA and WHO criteria. However, the cut-off level of FPG was slightly lower than that of the diagnostic criteria.

All three measures of glycaemia (FPG, 2-h PG and $\mathrm{HbA}_{1} \mathrm{c}$ ) were strongly associated with retinopathy in our Japanese population, which was similar to the findings in Pima Indians [3] and Egyptians [4], and to the results of the NHANES III [1]. The Egyptian study [4] reported that the area under the ROC curve for 2-h PG was similar to that for FPG, but that the area under the curve for $\mathrm{HbA}_{1} \mathrm{c}$ was significantly smaller than that for FPG and 2-h PG. The authors concluded that the FPG and 2-h PG were each strongly and equally associated with retinopathy for diagnostic purposes. In contrast, in the Pima Indian study [3], ROC analysis showed that the area under the curve for 2-h PG was slightly but not significantly larger than that for FPG and that for $\mathrm{HbA}_{1} \mathrm{c}$. This result is consistent with that of the present study. Our findings, together with those of the Pima Indian study, suggest that all measures are equally effective for diagnostic purposes, and that the FPG or $\mathrm{HbA}_{1} \mathrm{c}$ alone are also acceptable alternatives to $2-\mathrm{h}$ PG, which is complicated to measure by OGTT.

Optimal cut-off levels of plasma glucose for defining diabetes vary between populations. In the Pima Indian study [3], the ROC curve analysis identified the optimal FPG cut-off level as $6.8 \mathrm{mmol} / \mathrm{l}$. The NHANES III [1] also reported that the FPG cut-off level equivalent to the $2-\mathrm{h}$ PG criterion of $11.1 \mathrm{mmol} / \mathrm{l}$ was $6.7 \mathrm{mmol} / \mathrm{l}$. In contrast, we found that the optimal cut-off level of 2-h PG for diagnosis of diabetes, $11.1 \mathrm{mmol} / \mathrm{l}$, was consistent with current ADA and WHO criteria. However, the optimal cut-off level of FPG, $6.4 \mathrm{mmol} / \mathrm{l}$, was slightly lower than that of the diagnostic criteria [18]. Other Asian population studies $[19,20]$ have also reported that the optimal cut-off levels of FPG for diagnosis of diabetes ranged from 5.6 to $6.0 \mathrm{mmol} / \mathrm{l}$ in the sensitivity and specificity analysis for retinopathy. These findings suggest that the optimal level of diagnostic FPG is lower in Asian populations, including the Japanese population, than in western populations. In addition to the lower incidence of obesity in Asian populations, racial, genetic or environmental factors could contribute to this discrepancy.

This study has several limitations. Firstly, our results could be biased by the low participation rate. To ascer- tain the possibility of this bias, we compared the mean values of age, FPG, 2-h PG and $\mathrm{HbA}_{1}$ c levels as well as the proportion of men and women between the subjects who did participate in ophthalmic examination and those who did not. However, no significant differences in these parameters were observed between the groups, suggesting that this limitation does not invalidate the findings of the present study to a large extent. Secondly, our study population included individuals with diabetes who were taking oral anti-hyperglycaemic agents, resulting in a possible bias in the distribution of glycaemia. Although we performed analyses in which we excluded subjects on anti-hyperglycaemic medication, we could not draw any definitive conclusions due to the relatively low number of diabetic subjects not on antihyperglycaemic medication. However, it is to be expected that anti-hyperglycaemic medication has the same impact on FPG, 2-h PG and $\mathrm{HbA}_{1} \mathrm{c}$ levels, and thus medication was unlikely to have affected our findings on the ability of these measurements to predict diabetic retinopathy. In addition, we computed the FPG level equivalent of the 2-h PG criterion of $11.1 \mathrm{mmol} / \mathrm{l}$ among Hisayama residents who underwent OGTT in 1988 and found it to be $6.2 \mathrm{mmol} / \mathrm{l}$ (data not shown). This value was similar to the optimal FPG cut-off level of $6.4 \mathrm{mmol} / \mathrm{l}$ in the present study. It was therefore suggested that this type of bias did not distort the conclusions of our study. Thirdly, because of the cross-sectional design of this study, it is still unclear how the onset of retinopathy is related to the three measures of glycaemia. Further prospective investigation would help to clarify this issue.

In conclusion, after consideration of the risk of diabetic retinopathy, our population-based study suggests that measuring FPG or $\mathrm{HbA}_{1} \mathrm{c}$ is just as useful as measuring 2-h PG for the diagnosis of diabetes. Furthermore, there is a possibility that diabetic retinopathy occurs in patients with FPG levels lower than those currently used for the diagnosis of diabetes in the Japanese population.

\section{References}

1. The Expert Committee on the Diagnosis and Classification of Diabetes Mellitus (1997) Report of the Expert Committee on the Diagnosis and Classification of Diabetes Mellitus. Diabetes Care 20:1183-1197

2. World Health Organization (1999) Definition, diagnosis and classification of diabetes mellitus and its complications. Department of Noncommunicable Disease Surveillance, WHO, Geneva

3. McCance DR, Hanson RL, Charles MA et al. (1994) Comparison of test for glycated haemoglobin and fasting and two hour plasma glucose concentrations as diagnostic methods for diabetes. BMJ 308:1323-1328

4. Engelgau MM, Thompson TJ, Herman WH et al. (1997) Comparison of fasting and 2-h glucose and $\mathrm{HbA}_{1 \mathrm{c}}$ levels for diagnosing diabetes: diagnostic criteria and performance revisited. Diabetes Care 20:785-791 
5. Harris MI, Rowland M, Klein R, Byrd-Holt DD, Cowie CC (1998) Is the risk of diabetic retinopathy greater in non-Hispanic Blacks and Mexican Americans than in non-Hispanic Whites with type 2 diabetes? Diabetes Care 85:1230-1235

6. Harris EL, Sherman SH, Georeopoulos A (1999) Blackwhite differences in risk of developing retinopathy among individuals with type 2 diabetes. Diabetes Care 22:779-783

7. West SK, Klein R, Rodriguez J et al. (2001) Diabetes and diabetic retinopathy in a Mexican-American population. Diabetes Care 24:1204-1209

8. Ito C, Maeda R, Ishida S, Harada H, Inoue N, Sasaki H (2000) Importance of OGTT for diagnosing diabetes mellitus based on prevalence and incidence of retinopathy. Diabetes Res Clin Pract 49:181-186

9. Ohmura T, Ueda K, Kiyohara Y et al. (1993) Prevalence of type 2 (non-insulin-dependent) diabetes mellitus and impaired glucose tolerance in the Japanese general population: the Hisayama Study. Diabetologia 36:1198-1203

10. Ohmura T, Ueda K, Kiyohara Y et al. (1994) The association of the insulin resistance syndrome with impaired glucose tolerance and NIDDM in the Japanese general population: the Hisayama Study. Diabetologia 37:897-904

11. Miyazaki M, Nakamura H, Kubo M et al. (2003) Risk factors for age related maculopathy in a Japanese population: the Hisayama study. Br J Ophthalmol 87:469-472

12. Rajala U, Qiao Q, Laakso M, Keinänen-kiukaanniemi S (1998) Prevalence of retinopathy in people with diabetes, impaired glucose tolerance, and normal glucose tolerance. Diabetes Care 21:1664-1669
13. Diabetic Retinopathy Study Research Group (1981) Report 7: a modification of the Airlie House classification of diabetic retinopathy. Invest Ophthalmol Vis Sci 21:210-226

14. Klein R, Klein BEK, Magli YL et al. (1986) An alternative method of grading diabetic retinopathy. Ophthalmology 93:1183-1187

15. Early Treatment of Diabetic Retinopathy Study Research Group (1991) Report 10: grading diabetic retinopathy from stereoscopic fundus photographs: an extension of the Airlie House classification. Ophthalmology 98:786-806

16. Hanley JA, McNeil BJ (1982) The meaning and use of area under a receiver operating characteristic (ROC) curve. Radiology 143:29-36

17. Zweig MH, Campbell G (1993) Receiver-operating characteristic (ROC) plots: a fundamental evaluation tool in clinical medicine. Clin Chem 39:561-577

18. The Committee of the Japan Diabetes Society on the Diagnostic Criteria of Diabetes Mellitus (2002) Report of the Committee on the Classification and Diagnostic Criteria of Diabetes Mellitus. Diabetes Res Clin Pract 55:65-85

19. Lee C, Fook-Chong S (1997) Evaluation of fasting plasma glucose as a screening test for diabetes mellitus in Singaporean adults. Diabet Med 14:119-122

20. Nitiyanant W, Ploybutr S, Sriussadaporn S, Yamwong P, Vannasaeng S (1998) Evaluation of the new fasting plasma glucose cut-off level of $7.0 \mathrm{mmol} / 1$ in detection of diabetes mellitus in the Thai population. Diabetes Res Clin Pract 41:171-176 